\title{
Fuite ou accueil des cerveaux ? Une analyse de l'évolution du lieu de résidence des diplômés de l'Université de Bâle (2005)
}

Brain Drain or Brain gain? A study of destination of graduates of the University

of Basel (2005)

Brain Drain oder Brain Gain? Wohnorte von Absolventen der Universität Basel (2005)

\section{Benjamin Wieland}

\section{OpenEdition}

Journals

Édition électronique

URL : http://journals.openedition.org/rge/1756

DOI : $10.4000 /$ rge. 1756

ISSN : 2108-6478

Éditeur

Association des géographes de l'Est

Édition imprimée

Date de publication : 1 septembre 2008

ISSN : 0035-3213

\section{Référence électronique}

Benjamin Wieland, «Fuite ou accueil des cerveaux? Une analyse de l'évolution du lieu de résidence des diplômés de l'Université de Bâle (2005) », Revue Géographique de l'Est [En ligne], vol. 48 / 3-4 | 2008, mis en ligne le 02 mars 2010, consulté le 08 septembre 2020. URL : http://journals.openedition.org/ rge/1756 ; DOI : https://doi.org/10.4000/rge.1756

Ce document a été généré automatiquement le 8 septembre 2020

Tous droits réservés 


\title{
Fuite ou accueil des cerveaux? Une analyse de l'évolution du lieu de résidence des diplômés de l'Université de Bâle (2005)
}

\author{
Brain Drain or Brain gain? A study of destination of graduates of the University \\ of Basel (2005) \\ Brain Drain oder Brain Gain? Wohnorte von Absolventen der Universität Basel \\ (2005)
}

Benjamin Wieland

\section{Introduction}

1 Les sciences économiques s'accordent généralement à reconnaître que les pays industrialisés se dirigent vers une économie de la connaissance. Pour Drucker, qui a introduit le terme en allemand en 1969, cette évolution signifie que l'activité industrielle est de plus en plus souvent remplacée par des activités liées à la connaissance. Il s'ensuit que la pertinence de la formation augmente pour des couches plus larges de la population. En outre, la présence d'une main-d'œuvre hautement qualifiée constitue un facteur de localisation de plus en plus décisif pour les entreprises. Cette évolution est susceptible de conduire à des analyses géographiques intéressantes, en étudiant par exemple l'offre de main-d'œuvre disponible à l'échelle d'une région ou d'une nation. Pour cela, le « taux de maintien » constitue un indicateur général permettant de savoir si les diplômés d'une université ou d'une haute école, par exemple, ont trouvé un travail rémunéré dans leur région d'origine après l'aboutissement de leurs études.

2 La présente contribution analyse la proportion et la composition des diplômés de l'enseignement supérieur qui habitent encore ou qui sont revenus habiter dans le 
bassin d'emploi de Bâle cinq ans après avoir obtenu leur diplôme. Le bassin d'emploi considéré correspond quasiment à la Suisse du Nord-Ouest et comprend les cantons de Bâle-Ville, Bâle-Campagne, l'est du canton d'Argovie ainsi que la partie nord du canton de Soleure. Les données ont été récoltées au moyen d'un questionnaire de l'office fédéral de la statistique (OFS) adressé à 277 individus de l'Université de Bâle, qui représentent $34,8 \%$ des diplômés de l'année 2000 . La région de Bâle présente un taux de maintien de ses diplômés de $61,80 \%$ alors que la moyenne des douze universités suisses est de 51,07 \% dans leurs bassins d'emploi respectifs.

Sur les 102 diplômés de l'Université de Bâle ayant quitté leur bassin d'emploi cinq ans après avoir obtenu leur diplôme, ce qui peut être assimilé à une "fuite des cerveaux " ou brain drain, $83(81,4 \%)$ sont allés s'établir dans le reste de la Suisse et $19(18,6 \%)$ sont partis à l'étranger. Le bassin d'emploi de Zurich a le plus profité du départ des diplômés bâlois : parmi les diplômés précédemment décrits, 27 se sont établis dans la capitale économique de la Suisse. Dans le même temps, le bassin d'emploi de Bâle a bénéficié de l'apport de 68 diplômés en provenance des autres bassins du pays ou de l'étranger, ce qui peut être assimilé à un «accueil des cerveaux » ou brain gain selon son expression anglaise.

Les plus grandes différences en matière de taux de maintien s'observent généralement entre les différents domaines de compétence. C'est le cas également dans ce travail, comme le montrent les chiffres suivants qui présentent les taux de maintien des diplômés de l'Université de Bâle :

- Lettres, sciences humaines et sociales, théologie, psychologie: 77,2 \% (44 diplômés)

- Economie : 46,3 \% (19 diplômés)

- Droit : 69,2 \% (36 diplômés)

- Sciences naturelles : 52,5 \% (31 diplômés)

- Médecine : 60,3 \% (35 diplômés)

En résumé, les diplômés des lettres, sciences sociales et humaines, théologie, psychologie et droit montrent un attachement important à leur bassin d'origine. Pour les cantons qui ont consenti un investissement en faveur de ces individus, des gains peuvent par conséquent être attendus de ces individus, sous la forme d'impôts et de dépenses de consommation. En revanche, les étudiants des sciences économiques présentent une tendance beaucoup plus accentuée à quitter leur région d'origine, pour se diriger majoritairement vers le bassin d'emploi de Zurich.

\section{Etat de la littérature}

\section{A. Fondements théoriques de la mobilité des travailleurs hautement qualifiés}

6 La présente analyse s'appuie sur le constat que les pays industrialisés sont engagés dans une transformation conduisant à une « économie de la connaissance » dans laquelle la pertinence économique des travailleurs hautement qualifiés augmente (UNESCO 1995 ; OCDE 1996/2000). De nombreuses études ont pu mettre en évidence un lien fortement positif entre l'offre de capital humain et le développement économique (Becker 1964, Mankiw, Romer et Weil 1992 ; Murphy, Shleifer et Vishny 1991 ; Simon 1998). En outre, contrairement aux Etats-nations, il semble que les régions (re)gagnent une position forte en tant que structure spatiale de référence (Kulke 2004 : 194). Pour cette raison, la 
recherche d'une main-d'œuvre hautement qualifiée est devenue un objectif incontournable des politiques de promotion territoriale.

7 Il en est de même pour la région de Bâle, qui fait l'objet de la présente analyse et qui abrite un cluster d'entreprises travaillant dans le secteur de l'industrie chimique et pharmaceutique. L'activité de ces entreprises à haute intensité de connaissance, innovantes et globales, repose dans une large mesure sur des travailleurs hautement qualifiés (Dümmler et al. 2004:12/31; Credit Suisse 2004:52). Par conséquent, il incombe à la région de Bâle de faire la promotion de ses hautes écoles et de développer certaines formations adéquates pour faire en sorte que les diplômés qui sortent de ses institutions supérieures correspondent à la demande des entreprises et des organisations de la région.

8 Il importe de souligner que la présente étude ne cherche pas à connaître la composition actuelle ou l'origine des étudiants en se demandant par exemple qui est venu étudier à l'Université de Bâle. En revanche, la question centrale de l'analyse est de se demander si les diplômés de l'Université de Bâle habitent toujours dans la région où se sont déroulées leurs études et s'ils y ont trouvé une place de travail. Cela revient à se demander quelle est la part des diplômés de l'Université de Bâle qui sont restés dans la région et quels ont été leurs domaines de compétence.

\section{B. Etat de la recherche portant sur la mobilité des diplômés de l'enseignement supérieur en Suisse}

9 L'augmentation de la pertinence du capital humain a conduit à multiplier le nombre et l'intérêt des travaux portant sur ce sujet (Carnazzi et Golay 2005 ; Canton du Valais 2004 ; INTERREG IIIB NWE 2005). Contrairement aux migration en provenance des périphéries et des régions alpines, les mobilités qui s'établissent entre les centres urbains sont généralement désignées par le terme de brain exchange ou "échange de cerveaux", pour désigner qu'il s'agit d'un échange de main-d'œuvre hautement qualifiée. Bien que la plupart de ces échanges relèvent de la volonté des employeurs et soient limités dans le temps, ils conduisent néanmoins à un déficit de travailleurs pour l'une ou l'autre des régions, ce qui peut s'avérer problématique à moyen et à long terme (Meusburger 1998 : 380-383).

10 Dans son étude sur "La mobilité des jeunes hautement qualifiés en Suisse », l'office fédéral de la statistique (2007) a étudié la mobilité des diplômés ayant obtenu leur diplôme en 1998, 2000, 2002 et 2004. Au niveau géographique, les résultats peuvent être synthétisés de la manière suivante:

- Universités : les diplômés des cantons ne possédant pas de haute école connaissent une tendance à l'émigration supérieure à ceux dans lesquels se trouve un établissement d'enseignement supérieur.

- Entreprises à forte intensité de connaissances : il existe un lien entre la fuite des cerveaux et la répartition des entreprises à forte intensité de connaissances actives dans les domaines de la haute technologie et de la finance.

- Situation périphérique : elle concerne les cantons du Moyen Pays, des Grisons et du Valais, la Suisse orientale et centrale ainsi que les vallées du canton du Tessin.

- Centres urbains : les gagnants sont les villes universitaires de Bâle, Berne, Lausanne, Genève et Zurich et les communes de leurs agglomérations. Le canton de Zurich enregistre un bénéfice net de diplômés de $200 \%$. 
11 Selon les domaines de compétence des diplômés, il apparaît que les étudiants en droit connaissent une faible tendance à la mobilité, que les étudiants des sciences naturelles, des sciences économiques et des sciences techniques connaissent une tendance inverse marquée par une forte mobilité et que les étudiants des lettres et sciences sociales et humaines connaissent une tendance à l'émigration équilibrée.

\section{Méthodologie}

Les données utilisées dans cette analyse proviennent du deuxième questionnaire de l'OFS adressé aux diplômés en 2005. A cette époque, tous les diplômés de l'année 2000 qui avaient déjà participé à une première vague d'enquête en 2001 ont été interrogés une seconde fois. Selon l'OFS (2005 : 2-4), 123 diplômés ont été inclus en 1999 ou 2001. La seconde enquête de 2005 est exhaustive dans la mesure où les données des douze universités suisses sont disponibles.

13 Au total, 9575 personnes ayant achevé une licence, un diplôme, un master ou un bachelor sont concernées par le premier questionnaire adressé aux douze universités. A partir de cette population, 3285 diplômés ont répondu au second questionnaire envoyé en 2005. Sur les 794 diplômés, 277 ont répondu au questionnaire, ce qui correspond à un taux de réponse de $34,8 \%$.

\section{Résultats de l'analyse}

\section{A. Taux de maintien}

La ville de Bâle constitue le centre économique du bassin d'emploi bâlois. Ce bassin inclut notamment les cantons de Bâle-Ville et de Bâle-Campagne, le Fricktal (canton d'Argovie), ainsi que les districts de Dorneck et de Thierstein (canton de Soleure). Dans les analyses suivantes, les découpages cantonaux ne seront pas utilisés et seront remplacés par les régions de Mobilités Spatiales (MS), développées depuis 1982 pour servir d'unités d'analyse de l'emploi en Suisse. Certaines de ces régions MS s'étendent au-delà des frontières cantonales, comme la MS-25 de la région du Laufental, située à cheval sur les cantons de Bâle-Campagne et de Soleure. Les bassins d'emploi s'appliquent aux trajets pendulaires de 16 centres de moyenne et grande taille depuis 1960.

La région de Bâle se limite au territoire suisse, ce qui implique que les diplômés habitant les communes sud-est du département français du Haut-Rhin ainsi que les communes allemandes du Kreis de Lörrach ne sont pas pris en considération et sont considérés comme des personnes vivant à l'étranger. 
Carte 1 : Les 106 régions MS et les 16 bassins d'emploi de Suisse

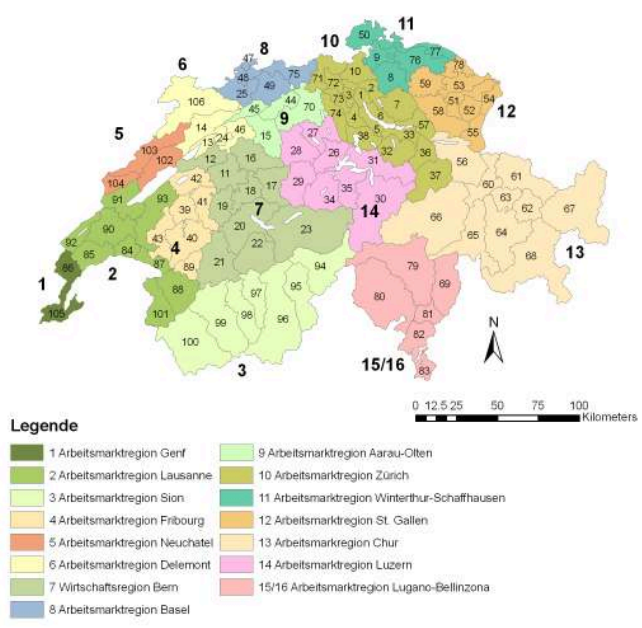

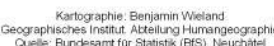

Source : OFS 2005

Tableau 1 : Rang des hautes écoles universitaires selon le taux de maintien

\begin{tabular}{|l|c|c|c|c|}
\hline Hautes écoles universitaires & $\begin{array}{c}\text { Bassin } \\
\text { d'emploi }\end{array}$ & $\begin{array}{c}\text { Taux de maintien } \\
\text { (\%) }\end{array}$ & Rang & $\begin{array}{c}\text { Réponses } \\
\text { valides }\end{array}$ \\
\hline $\begin{array}{l}\text { Université de la Suisse } \\
\text { italienne }\end{array}$ & $15 / 16$ & 84,00 & $\mathbf{1}$ & 25 \\
\hline Université de Genève & 1 & 69,07 & $\mathbf{2}$ & 375 \\
\hline Université de Zurich & 10 & 69,06 & $\mathbf{3}$ & 682 \\
\hline Université de Bâle & 8 & 61,80 & $\mathbf{4}$ & 267 \\
\hline Université de Lausanne & 2 & 61,74 & $\mathbf{5}$ & 298 \\
\hline EPF de Zurich & 10 & 53,48 & $\mathbf{6}$ & 417 \\
\hline Université de Berne & 7 & 50,24 & $\mathbf{7}$ & 412 \\
\hline \hline Université de Lucerne & 14 & 44,44 & $\mathbf{8}$ & 9 \\
\hline EPF de Lausanne & 2 & 37,66 & $\mathbf{9}$ & 154 \\
\hline Université de Neuchâtel & & $\mathbf{1 0}$ & 118 \\
\hline
\end{tabular}




\begin{tabular}{|l|c|c|c|c|}
\hline Université de Fribourg & 4 & 25,00 & $\mathbf{1 1}$ & 252 \\
\hline \hline Université de Saint-Gall & 12 & 21,54 & $\mathbf{1 2}$ & 130 \\
\hline Total/Moyenne & - & $\mathbf{5 1 , 0 7}$ & - & $\mathbf{3 1 3 9}$ \\
\hline Université et EPF de Zurich & 10 & 63,15 & - & 1099 \\
\hline Université et EPF de Lausanne & 2 & 53,54 & - & 452 \\
\hline
\end{tabular}

Source des données : OFS 2005

Le tableau présente les résultats du " classement » effectué sur les hautes écoles selon leur taux de maintien. Il est important de noter que deux établissements d'enseignement supérieur sont situés dans la région MS-10 (Zurich) et MS-2 (Lausanne). Les deux Ecoles Polytechniques Fédérales (EPF) affectent le bassin d'emploi de leur région respective. L'EPF de Zurich polarise une partie de la Suisse de langue allemande alors que l'EPF de Lausanne s'adresse à la partie francophone. Il est néanmoins judicieux de séparer les deux EPF de chaque région d'analyse. Le tableau suivant présente les taux de maintien des quatre établissements d'enseignement supérieur du marché du travail des régions de Zurich et de Lausanne. Les résultats obtenus par le calcul des taux permettent de dresser le classement suivant:

Figure 1 : Lieu de résidence des diplômés de l'Université de Bâle à l'âge de 16 ans, pendant la durée des études, au cours de la première activité et cinq ans après l'obtention du diplôme

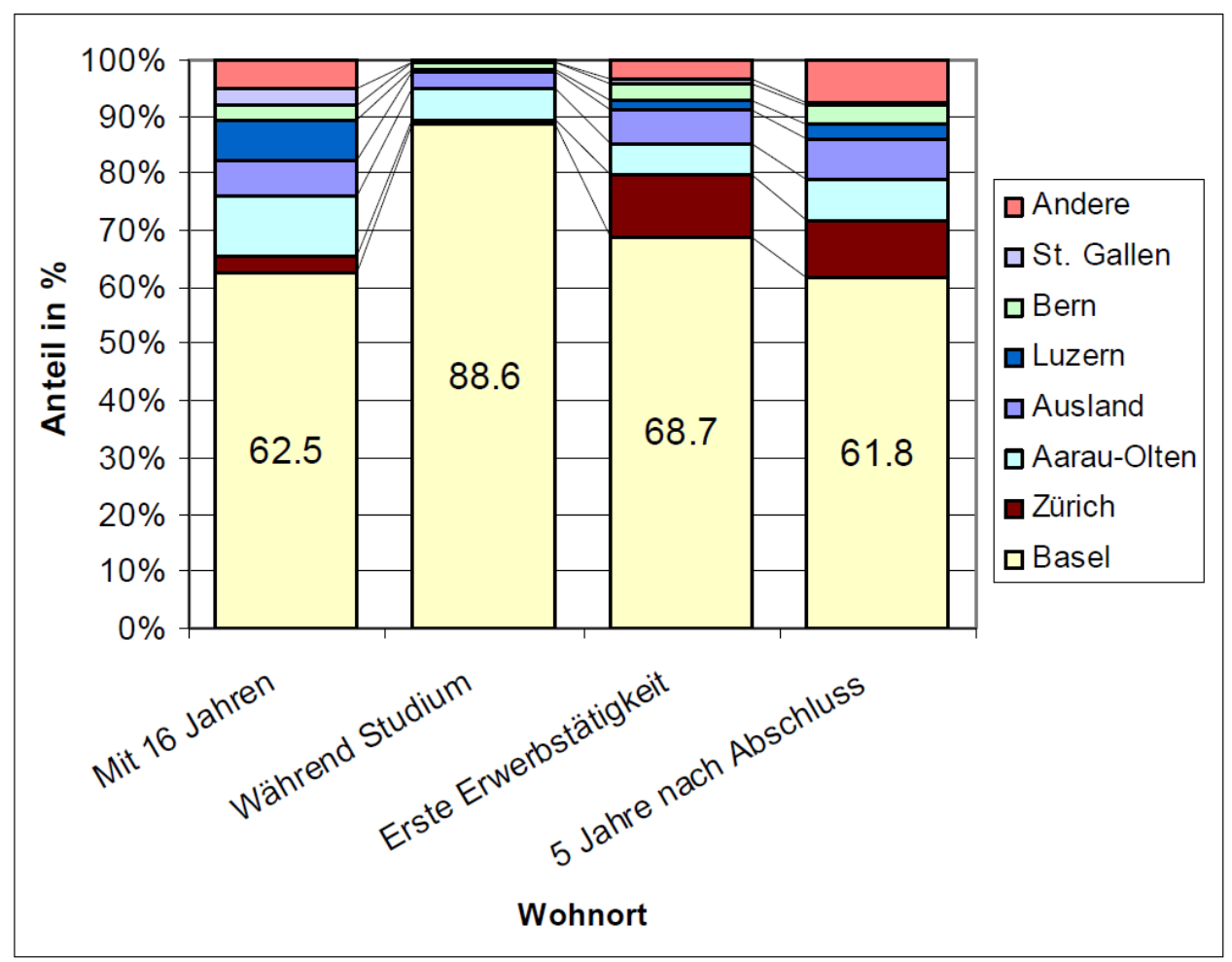

Source des données: OFS 2005 
17 Le graphique montre l'évolution du lieu de résidence des diplômés de l'Université de Bâle par bassins d'emplois. La proportion de diplômés domiciliés dans le bassin d'emploi de Bâle diminue de 1,3\% (-20 élèves) entre l'âge de 16 ans et cinq ans après l'obtention du diplôme. Il est frappant de constater la part élevée des diplômés qui résident dans le bassin de Bâle pendant la durée des études (88,6\%). Après la fin des études, la part du bassin de Zurich augmente fortement.

Cartes 2 et 3 : Lieu de résidence des diplômés de l'Université de Bâle à l'âge de 16 ans et pendant la durée de leurs études

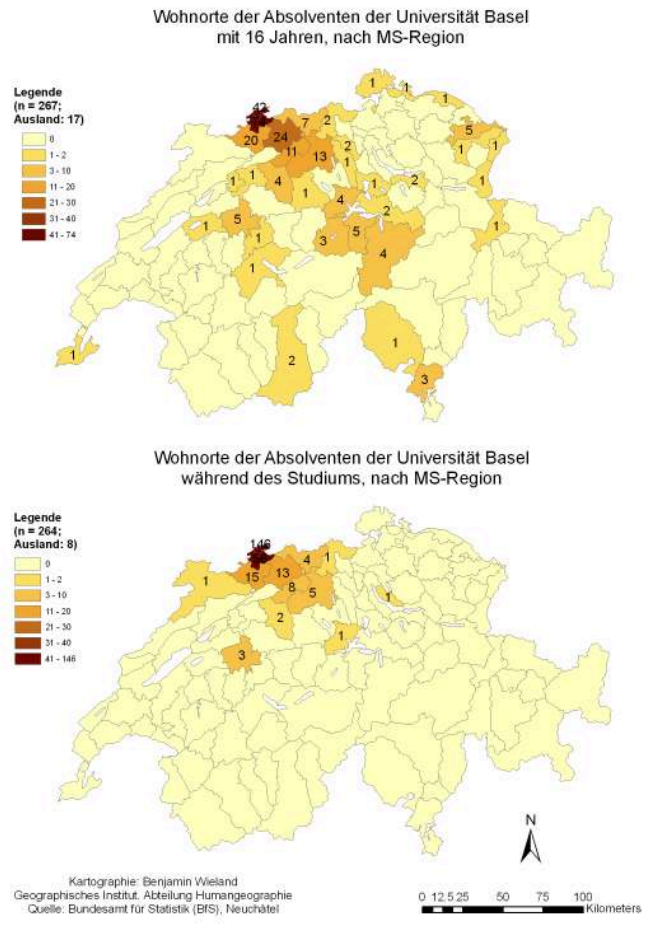

Source des données : OFS 2005 
Cartes 4 et 5 : Lieu de résidence des diplômés de l'Université de Bâle au début de la première activité et cinq ans après l'obtention de leur diplôme

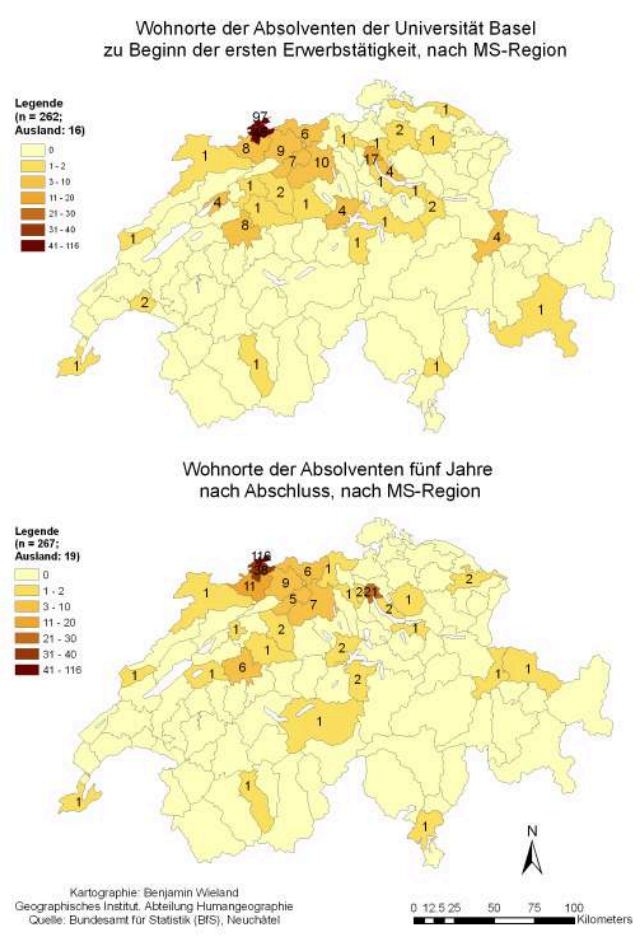

\section{B. Comparaison des émigrants et destination selon les disciplines}

Afin d'approfondir l'analyse, les groupes composés des diplômés ayant émigrés et de ceux ayant choisi de rester sur place sont comparés selon leurs domaines de compétence. Le tableau suivant présente les résultats de cette comparaison (les réponses non valides sont omises) :

Tableau 2 : Taux de maintien des diplômés selon le domaine de compétence

\begin{tabular}{|l|c|c|c|}
\hline Domaines de compétence & $\begin{array}{c}\text { Diplômés demeurés } \\
\text { sur place }\end{array}$ & $\begin{array}{c}\text { Diplômés ayant } \\
\text { émigrés }\end{array}$ & Total \\
\hline $\begin{array}{l}\text { Lettres, sciences sociales et humaines, } \\
\text { psychologie, théologie }\end{array}$ & $44^{*}$ & $13^{*}$ & 57 \\
\hline Economie & $19^{* *}$ & $22^{* *}$ & 41 \\
\hline Droit & 36 & 16 & 52 \\
\hline Sciences naturelles & $31^{*}$ & $28^{*}$ & 59 \\
\hline Médecine & 35 & 23 & 58 \\
\hline
\end{tabular}




\begin{tabular}{|l|c|c|c|}
\hline Total & 165 & 102 & 267 \\
\hline
\end{tabular}

** significatif à $1 \%$; * significatif à $5 \% ; \mathrm{p}<0,01$; Cramers $V=0,233$

Source des données : OFS 2005 ; calculs de l'auteur

Figure 2 : Proportion des diplômés de l'Université de Bâle ayant choisi d'émigrer ou de demeurer sur place selon le domaine de compétence

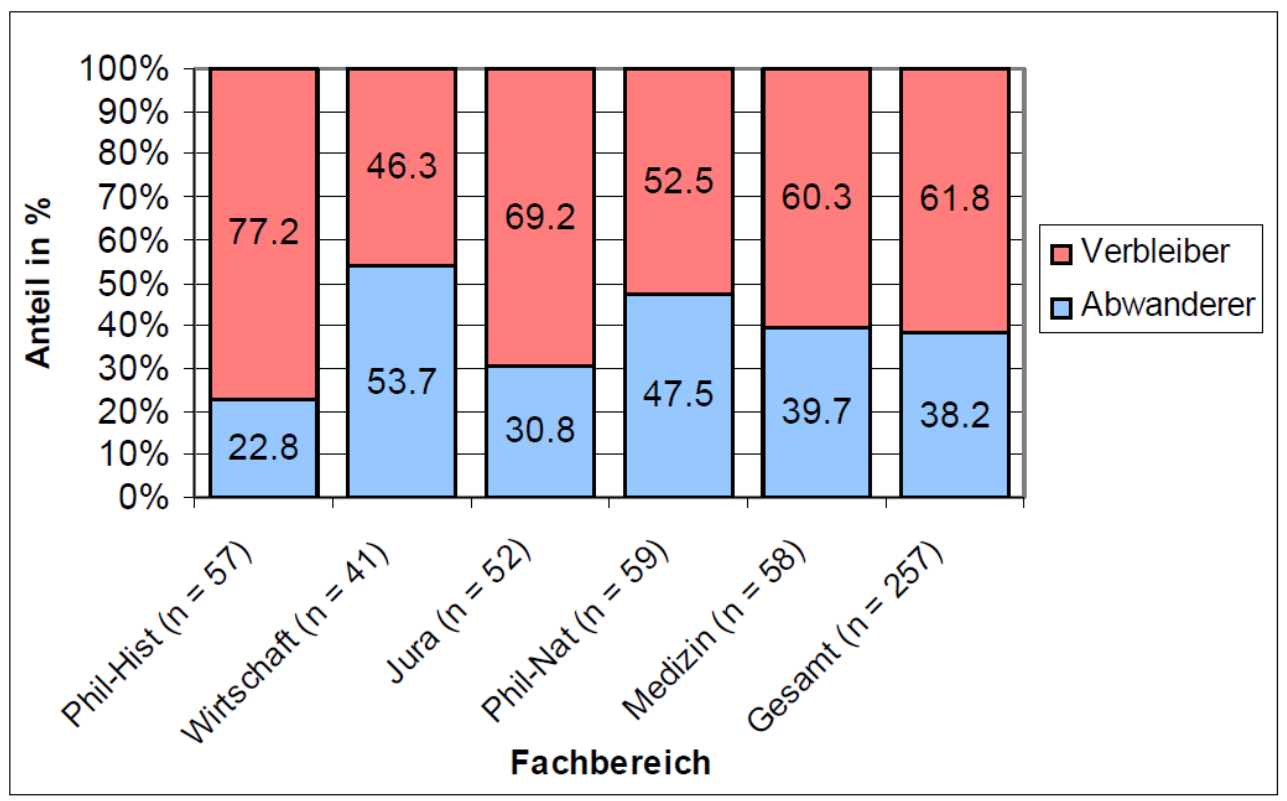

Source des données : OFS 2005 ; calculs de l'auteur

Le graphique illustre les différences qui existent entre les diplômés de l'Université de Bâle qui ont choisi de changer de bassin d'emploi et les autres, en fonction des domaines de compétence.

En Suisse, les plus grandes différences en matière de mobilité peuvent généralement être observées entre les domaines de compétence. L'analyse montre que c'est aussi le cas des diplômés de l'Université de Bâle. Le plus haut taux de maintien concerne les diplômés des sciences humaines et sociales, de psychologie et de théologie $(77,2 \%)$. La plus forte mobilité territoriale concerne les économistes, dont un peu plus de la moitié (53,7 \%) n'habitent plus le bassin d'emploi de Bâle cinq ans après l'obtention de leur diplôme. Bien que des emplois soient disponibles dans la région de Bâle, les entreprises actives dans le domaine des services financiers situées dans le bassin de Zurich exercent une forte attraction sur les jeunes diplômés.

Une attention particulière doit être accordée aux diplômés des sciences naturelles, car ils sont particulièrement nombreux et demandés dans les entreprises et les organisations du cluster des sciences de la vie de l'agglomération trinationale de Bâle. Ces jeunes diplômés des sciences naturelles connaissent également un fort désir d'émigrer : près de la moitié n'habitent plus la Suisse du Nord-Ouest cinq ans après l'obtention de leur diplôme (28, soit 47,5\%). 


\section{Discussion des résultats et conclusion}

l'Université de Bâle forment une main-d'œuvre demandée et concurrentielle sur le marché du travail de la Suisse du Nord-Ouest. Le bassin d'emploi de Bâle a pour particularité de compter une forte proportion de diplômés désireux de rester là où ils se trouvent actuellement par rapport aux diplômés des autres universités de Suisse (61,80 \%). Il importe de noter également que certaines parties du bassin bâlois n'ont pas été prises en considération. Si les diplômés venus s'établir dans le Kreis de Lörrach ou dans le sud de l'Alsace cinq ans après l'obtention de leur diplômes avaient été considérés comme relevant du bassin de Bâle, alors le taux de maintien serait vraisemblablement plus élevé de quelques pourcents.

Selon les autorités universitaires des cantons bâlois, l'Université de Bâle doit renforcer les sciences de la vie et les formations liées à la culture déjà existantes et conserver un large éventail de possibilités (Rektorat und Ressort öffentlichkeitsarbeit der Universität Basel 2006: 15; Strategiegruppe Life Sciences 2007). Les résultats de cette étude peuvent être considérés à la lumière de ces deux exigences contradictoires adressées à l'université. D'une part, il semble que l'Université de Bâle joue son rôle de soutien régional au savoir, notamment dans les domaines qui relèvent de la culture, comme les lettres et les sciences humaines et sociales. D'autre part, il semble que le bilan soit plus mitigé dans le domaine des sciences de la vie. Parmi les diplômés des sciences naturelles, comme nous l'avons déjà souligné, un peu plus de la moitié habitent encore le bassin d'emploi de Bâle cinq ans après l'obtention de leur diplôme (52,4\%). Dans ce domaine, l'objectif devrait être d'attirer des individus talentueux d'Europe et du monde entier afin qu'ils effectuent leurs études à Bâle.

En conclusion, il convient de se demander si les deux tâches contradictoires adressées à l'Université de Bâle que sont la recherche de pointe dans un certain nombre de domaines et le soutien régional au savoir pourront être financées à long terme, compte tenu de la hausse des effectifs. Il semble que l'université pourrait concentrer ses efforts dans les matières pour lesquelles un marché régional dynamique est disponible pour les jeunes diplômés. Pour les domaines qui ne remplissent pas cette condition, des économies pourraient être prévues. Les résultats de la présente étude indiquent que les sciences économiques sont particulièrement concernées par ce dernier point, du fait qu'une importante proportion de la force de travail universitaire est attirée par le canton de Zurich à l'issue de ses études à Bâle.

BIBLIOGRAPHIE

Becker G.S. (1975 [1964]). - Human Capital: A Theoretical and Empirical Analysis, with Special Reference to Education, seconde édition, New York, National Bureau of Economic Research. 
Office fédéral de la statistique OFS (2007). - Regionale Abwanderung von jungen Hochqualifizierten in der Schweiz. Empirische Analyse der Hochschulabsolventenjahrgänge 1998 bis 2004, Neuchâtel.

Office fédéral de la statistique OFS (2005a). - Schweizer Teilstudie zum Projekt The Flexible Professional in the Knowledge Society. Projektinformationen, Neuchâtel.

Office fédéral de la statistique OFS (2005b). - 106 MS-Regionen der Schweiz. Les 106 régions MS de la Suisse, Neuchâtel.

Carnazzi, S.; Golay, S. (2005). - Interne Migration in der Schweiz. Eidgenössische Volkszählung 2000, Zurich, Neuchâtel, Crédit Suisse Economic Research \& Bundesamt für Statistik - http:// www.bfs.admin.ch/bfs/portal/de/index/infothek/publ.Document.64467.pdf

Crédit Suisse (2004). - Wirtschaftsraum Basel. Struktur und Perspektiven, Zurich.

Drucker, P. F. (1969). - Die Zukunft bewältigen. Aufgaben und Chancen im Zeitalter der Ungewissheit, Düsseldorf, Econ.

Dümmler, P.; Abegg, Ch.; Kruse, Ch.; Thierstein, A. (2004). - Analysen zur Betriebszählung 2001: Standorte der innovativen Schweiz: Räumliche Veränderungsprozesse von High-Tech und Finanzdienstleistungen, Neuchâtel, office fédéral de la statistique.

INTERREG IIIb NWE (2005). - Human capital in European peripheral regions: Brain-Drain and Brain-Gain - http://www.brain-drain.org/index_html

Canton du Valais (2004). - Wegziehen - bleiben - zurückkehren. Eine wissenschaftliche Studie der Arbeitsmarktbeobachtung Wallis zur Abwanderung von Kompetenzen von Walliserinnen und Wallisern, Sion, Département de l'économie, des institutions et de la sécurité, Département de l'éducation, de la culture et du sport.

Kulke, E (2004). - Wirtschaftsgeographie, Paderborn, Schöningh.

Mankiw, G., Romer, D., Weil, D. (1992). - « A contribution to the empirics of economic growth »: Quarterly Journal of Economics, ${ }^{\circ} 107$, p.407-437.

Murphy, K. M., Shleifer, A., Vishny R. (1991). - « Allocation of talent: Implications for growth », Quarterly Journal of Economics, ${ }^{\circ} 106$, p.503-530.

Meusburger, P. (1998). - Bildungsgeographie: Wissen und Ausbildung in der räumlichen Dimension, Heidelberg, Spektrum.

Organisation for Economic Co-Operation And Development OECD (2000). - OECD STI Outlook 1996. Outlook of the Directorate of Science, Technology and Industry, Paris, OECD publication service.

Organisation for Economic Co-Operation And Development OECD (1996). - The Knowledge-based economy, Paris, OECD publications service.

Rektorat und Ressort Öffentlichkeitsarbeit der Universität Basel (2006). - Strategie 2007. Zur Entwicklung der Universität Basel 2007-2013, Bâle.

Simon, C. (1998). - Human capital and metropolitan employment growth, Journal of Urban Economics, ${ }^{\circ} 43 / 2$, p.223-243.

Strategiegruppe «Life Sciences » (2007). - Forschungsstrategie der Universität Basel im Profilierungsbereich «Life Sciences », Thesenpapier.

UNESCO (1995). - Towards Knowledge Societies. United Nations Educational, Scientific and Cultural Organization, Paris, UNESCO Publishing. 


\section{RÉSUMÉS}

Cet article analyse le taux de maintien des diplômés de l'Université de Bâle et s'interroge sur le lien entre le lieu où ces diplômés ont effectué leurs études et leur choix de résidence. La base de données utilisée repose sur un questionnaire de l'office fédéral de la statistique suisse administré en 2005 à 277 participants. L'enquête a été effectuée en moyenne cinq ans après l'obtention du diplôme. L'espace de référence est constitué par le bassin d'emploi régional de Bâle. L'analyse du choix de résidence montre que 61,8\% des diplômés de l'Université de Bâle habitent toujours dans le bassin d'emploi du même nom. La plus grande partie des 102 diplômés ayant décidé de quitter ce bassin, qui illustrent une forme de " fuite des cerveaux » (ou brain drain), se sont dirigés vers le bassin d'emploi de Zurich. Les principales différences relevées se rapportent aux champs de compétence des diplômés. Ainsi, les jeunes universitaires issus des sciences humaines et sociales, de la théologie et de la psychologie présentent un taux de maintien de $77,2 \%$, contre seulement $46,3 \%$ pour ceux qui ont étudié l'économie.

This article analyses the destination of graduates of the University of Basel, Switzerland. Particular attention is paid to possible links between choice of studies and eventual destination. Use is made of data collected by the Federal Statistical Office in 2005 in a survey of 277 persons about five years after graduation. Basel is defined here as the labour market area of Basel. Results indicate that $61.8 \%$ of survey participants were still living in the Basel five years after graduation. Of the 102 graduates that left the region («Brain Drain»), the majority had moved to the labour market area of Zurich. The biggest difference concerning destination was to be found between study areas. $77.2 \%$ of graduates of the humanities, social sciences, theology and psychology stayed in the region, in comparison to only $46.3 \%$ of economic graduates.

Der vorliegende Beitrag untersucht die Verbleibsquote von Absolventen der Universität Basel und geht der Frage nach, ob zwischen Studium und Wohnortwahl ein Zusammenhang besteht. Als Datengrundlage dient ein Fragebogen des Bundesamtes für Statistik aus dem Jahr 2005 mit 277 Teilnehmern. Die Befragung erfolgte durchschnittlich fünf Jahre nach Studienabschluss. Als räumliche Abgrenzung dient die so genannte Arbeitsmarktregion Basel. Die Analyse der Wohnorte ergab, dass $61.8 \%$ der Universität-Basel-Absolventen innerhalb der Arbeitsmarktregion Basel wohnen. Von den insgesamt 102 abgewanderten Absolventen («Brain Drain») war der grösste Teil in der Arbeitsmarktregion Zürich wohnhaft. Die grössten Unterschiede beim Verbleib ergaben sich zwischen den einzelnen Fachbereichen. Absolventen der Geistes- und Sozialwissenschaften, der Theologie und Psychologie weisen eine Verbleibsquote von $77.2 \%$ auf - der geringste Wert lässt sich mit $46.3 \%$ bei den Wirtschaftswissenschaften feststellen.

\section{INDEX}

Mots-clés : délocalisations, diplômés, fuite des cerveaux, société de la connaissance, taux de maintien, université

Schlüsselwörter : Abwanderung, Brain Drain, Hochschulabsolventen, Universität, Verbleibsquote, Wissensgesellschaft

Keywords : brain drain, graduate destination profiles, graduate movement, knowledge society, university, university graduates 
AUTEUR

BENJAMIN WIELAND

Drahtzugstrasse 36, 4057 Bâle - benjamin.wieland@baz.ch 